\title{
Trabajo Infantil y Subjetividad: una perspectiva necesaria
}

\author{
Albertina Mitjáns Martinez. \\ Universidade de Brasília
}

\begin{abstract}
Resumen
A partir de la línea teórica que, dentro del enfoque histórico-cultural ha desarrollado el concepto de subjetividad, se discute la pertinencia y la necesidad del estudio de la constitución y del desarrollo de la subjetividad de los niños y adolescentes trabajadores. También se fundamenta el valor heurístico de las categorías subjetividad, personalidad y sujeto para la compreensión del impacto del trabajo precoz en el desarrollo psicológico. Se presentan los resultados de un estudio exploratório realizado com adolescentes trabajadores que muestran cuatro formas diferenciadas de subjetivación de la situación de trabajo, hecho que puede condicionar trayectorias diferenciadas en el desarrollo de la subjetividad. A partir de estos resultados se cuestiona el significado de algunas afirmaciones generales que aparecen con frecuencia en la literatura especializada sobre las consecuencias del trabajo infantil en el desarrollo psicológico. Por último se muestra que la posiblidad de profundizar en la compreensión de la constitución de la subjetividad puede contribuir a la elaboración de estratégias educativas diferenciadas y potencialmente, más efectivas en el trabajo con esta población.

Palabras clave: Subjetividad, Trabajo infantil, Enfoque histórico-cultural, Desarrollo infantil.
\end{abstract}

\section{Resumo}

Trabalho infantil e subjetividade: uma perspectiva necessária. A partir da linha teórica, que dentro do enfoque histórico-cultural tem desenvolvido a concepção da subjetividade, discute-se tanto a pertinência quanto a necessidade do estudo da constituição e do desenvolvimento da subjetividade individual das crianças e dos adolescentes trabalhadores e fundamenta-se o valor heurístico das categorias subjetividade, personalidade e sujeito na compreensão do impacto do trabalho precoce no desenvolvimento psicológico. São apresentados os resultados de um estudo exploratório realizado com adolescentes trabalhadores que mostra quatro formas diferenciadas de subjetivação da situação de trabalho, fato que pode condicionar trajetórias diferenciadas no desenvolvimento da subjetividade. A partir destes resultados questiona-se o significado de algumas afirmações gerais que aparecem com freqüência na literatura especializada sobre as conseqüências do trabalho infantil no desenvolvimento psicológico. Por último, mostra-se que a possibilidade de aprofundar na compreensão da complexidade da constituição da subjetividade pode contribuir para a elaboração de estratégias educativas diferenciadas e potencialmente mais efetivas, no trabalho com esta população.

Palavras-chave: Subjetividade, Trabalho infantil, Enfoque histórico-cultural, Desenvolvimento infantil.

\begin{abstract}
Child work and subjectivity: A necessary approach. On the basis of theoretical framework oriented towards the topic of subjectivity within cultural-historical approach the pertinence and need of the study and development of individual subjectivity of children working are discussed, emphasizing the heuristic value of the categories of subjectivity, personality, and subject in the comprehension of the impact of child work on psychological development. The results of an exploratory study with adolescent workers are presented, in which four differentiating ways of subjectivization of work activity clearly
\end{abstract}


appear. This fact may also influence on differentiating ways in the development of subjectivity. Based on these results the meaning of certain general assumptions related to the consequences of child work on psychological development is questioned. These assumptions frequently appear in specialized literature in relation to this topic. Finally, it is suggested that a better and deep knowledge about the complexity of subjective constitution of the subject may contribute to the development of differentiating educational strategies, potentially more effective, in order to educate this population.

Key words: Subjectivity, Child work, Cultural-historical Approach, Child development

\section{La dimensión psicológica del trabajo infantil}

$\mathrm{E}$ 1 trabajo infantil ${ }^{1}$, como dura realidad que afecta a decenas de países, constituye un objeto de investigación abordado desde diferentes ángulos y perspectivas. Creciente atención ha merecido el estudio de las consecuencias del trabajo infantil en los planos económico y social y especialmente, las consecuencias que este fenómeno tiene para la salud y para la escolarización de los niños y adolescentes que trabajan (Campos, Dimenstein \& Francischini, 2000).

Es reconocido que la investigación de las consecuencias del trabajo infantil, por su complejidad, demanda de la participación de diferentes campos disciplinares, donde cada uno de ellos contribuya, en su especificidad y en su articulación con los restantes, a la comprensión del objeto de estudio (Ferreira, Silva \& Hoelzel, 2000). Así, el objetivo principal de este artículo es analizar y fundamentar lo que consideramos una de las contribuciones que la Psicología, como ciencia particular, puede hacer al estudio de las consecuencias del trabajo infantil: el análisis del impacto de la situación de trabajo en la constitución y desarrollo de la subjetividad de los niños y adolescentes que trabajan.

Nos parece necesario desarrollar esta línea de trabajo, no sólo por el valor que puede representar desde el punto de vista teórico para la comprensión del funcionamiento de la subjetividad humana, sino, fundamentalmente, por su valor para el diseño diferenciado de estrategias de intervención educativas y comunitarias, potencialmente más efectivas.

La Psicología, como ciencia particular, no ha tenido una presencia relevante con relación a la investigación del trabajo infantil y de sus consecuencias; la arista psicológica de este complejo problema ha sido, relativamente, mucho menos trabajada que otros aspectos como el económico, el jurídico y el social. A nuestro modo de ver, esto no es sólo explicable por el hecho de que la gravedad del trabajo infantil, como fenómeno social, y la necesidad de elaborar políticas públicas, imponen prioridades a la investigación, proceso en el que algunas disciplinas se evidencian como más pertinentes que otras. Hipotetizamos que existen, también, otras razones, relativas a la propia Psicología, que pudieran contribuir a comprender este hecho.

Una de ellas, a nuestro juicio, es que la mayor parte de la producción científica en Psicología ha estado relacionada, fundamentalmente, con problemas y cuestiones que emergen como importantes en los grandes polos de producción de conocimiento científico: Europa y Estados Unidos. Los temas que no forman parte de las principales corrientes de producción de conocimiento, por estaren relacionados a fenómenos que caracterizan otros contextos socioeconómicos, como el trabajo infantil, entre otros, tienen mucha más dificultad en constituirse y legitimarse como campos de investigación y de producción teórica.

Por outra parte, la Psicología, históricamente, se ha caracterizado por su fragmentación (García Rosas, 1973; González Rey, 1989, 1995, 1997; González Rey \& Mitjáns Martínez, 1989). Una de sus formas de expresión ha sido la constitución y el desarrollo de áreas o campos de trabajo con objetos de estudio específicos, así como con categorías y sistemas de conocimiento propios, sin una articulación real entre ellos. Esto dificulta el abordaje de problemas que se situan en las interfases de esas áreas tradicionales o que precisan de nuevos marcos para su compreensión. Las consecuencias del trabajo infantil para el desarrollo psicológico, por ejemplo, constituye un problema que se ubica, esencialmente, en lainterfase de lo que tradicionalmente sería la Psicología del Desarrollo y la Psicología del Trabajo demandando una visión y una formación, que incluya, al menos en sus aspectos esenciales, sistemas de conocimientos correspondientes a las dos áreas. A nuestro modo de ver, la complejidad de muchos de los temas referidos al impacto, que en el plano subjetivo tiene el trabajo infantil, demanda la utilización creativa e simultánea de cuerpos de conhecimentos que aparecen hoy fragmentados y asociados a diferentes áreas de la Psicologia como Psicologia Escolar, del Desarrollo, del Trabajo, Familiar y de la Salud, fundamentalmente. Esta visión de articulación e integración del conocimiento psicológico no ha sido una posición dominante en la Psicología al abordar objetos de estudio complejos, 
lo que ha limitado el desarrollo de la producción científica en muchos temas, entre ellos la dimensión psicológica del trabajoinfantil.

Otra razón que deve considerarse es la relativa a los problemas existentes en la formación de los profesionales de la Psicologia, formación casi siempre acrítica, centrada en la reproducción de los sistemas de conocimientos y modelos de acción dominantes, en la cual, la creatividad, la función social de la profesión y la sensiblidad ante los problemas sociales, no son cuestiones suficientemente priorizadas y trabajadas. La dedicación al estudio del trabajo infantil implica una sensiblidad social y una postura crítica y comprometida que no constituyen objetos de trabajo educativo intencional en la mayoría de las instituciones formadoras de psicólogos, lo que no favorece que un mayor número de profesionales preparados se vinculen a este campo de trabajo.

A todo lo anterior se suman las dificultades teóricas y metodológicas que el propio objeto de investigación plantea. El estudio de los niños y adolescentes que trabajan supone nuevos desafíos teóricos, entre otras cosas, porque se desarrollan en un contexto diferente a los contextos donde se han realizado la mayoría de las investigaciones y se ha producido gran parte del conocimiento en relación con el desarrollo infantil y del adolescente, como la familia y la escuela. Metodológicamente, trabajar con este tipo de sujetos impone también situaciones nuevas, ya que algunos de los instrumentos y formas de trabajo que se utilizan com niños y adolescentes en otras condiciones, tienen que ser reformulados creativamente para trabajar con esta población, la que también demanda formas diferenciadas de aproximación e indagación.

Creemos que la Psicología puede aumentar su espacio en la investigación sobre el trabajo infantil y una de las posibilidades para ello es la introducción de la perspectiva de la subjetividad, perspectiva que no ha sido suficientemente explorada, a mi juicio, por otras razones adicionales a las anteriormente expuestas.

En el abordaje socio-psicológico del trabajo infantil ha predominado un enfoque "macro" que, desde mi punto de vista, está inserido en el carácter fundamentalmente descriptivo y cuantitativo que ha dominado gran parte de la investigación psicológica. Este tipo de investigación tiene el valor de describir el fenómeno en sus aspectos más generales y, de hecho, ser un espacio de generación de hipótesis para momentos más explicativos del conocimiento, sin embargo tiene la limitación de centrarse en aquello que aparece con más frecuencia, a partir de lo cual se realizan generalizaciones que no permiten mostrar la diversidad y complejidad del fenómeno real. En este tipo de investigación, el sujeto concreto, el niño y el adolescente que trabaja, queda perdido, subsumido en lo que aparece con más frecuencia en todos ellos. En la Psicología esta perspectiva ha resultado dominante por muchos años y no ha favorecido, en sus diferentes campos, un análisis más complejo orientado a la comprensión del funcionamiento de la subjetividad individual.

Sin menospreciar el valor que los estudios de corte descriptivo-cuantitativo tienen para el análisis del trabajo infantil, queremos fundamentar la necesidad de estudios que rescaten el sujeto que trabaja, estudios que permitan contribuir a comprender las complejas y variadas formas en que el trabajo los constituye. Un tipo de investigación que se inscribe en esta dirección es por ejemplo, el trabajo realizado por Bonamigo (1996) sobre la construcción de la identidad en adolescentes trabajadores.

Coincidimos con Marques (1998) cuando, con relación a la realidad brasilera, afirma: "ya que el trabajo ha aparecido en la vida de un conjunto significativo de niños de nuestra población, nos corresponde, como cientistas sociales, buscar la comprensión de las consecuencias de esa realidad para la subjetividad de los grupos sociales que están sometidos a ese proceso" (p. 51). Indiscutiblemente, ésta es una tarea que le corresponde a la Psicología dentro del conjunto de ciencias que estudian este complejo fenómeno y contribuiría a las transformaciones que están indicando Ferreira, Silva y Hoelzel (2000) con relación a la evolución de la investigación del trabajo infantil al afirmar:

La experiencia de los grupos de investigación brasileros con relación al trabajo precoz va poco a poco superando el examen de los aspectos múltiples y genéricos referentes al tema de la inserción precoz en el trabajo, bajo una óptica donde predomina el abordaje tipo survey, para profundizar en recortes específicos, en realidades micro que exigen el concurso de especialistas de varias áreas (p. 59).

Coincidimos con la necesidad del trabajo interdisciplinario, especialmente, si los recortes investigativos respetan y aprehenden la complejidad del objeto de estudio. Sin embargo, no podemos perder de vista que, sin un desarrollo fuerte de un campo disciplinar, tanto desde el punto de vista epistemológico, como teórico y metodológico, no es posible pensar en un trabajo interdisciplinario efectivo (González Rey, 1993). La Psicología puede y debe fortalecer su propio espacio en la investigación sobre el trabajo infantil so pena de ser subsumida por otras ciencias y lo que es más importante, corriendo el riesgo de no contribuir, desde su cosmovisión, al trabajo interdisciplinario que demanda la complejidad e multiplicidad de ese fenómeno.

Fortalecer la participación de la Psicología en la investigación sobre el trabajo infantil, trabajando en perspectivas que rescaten el sujeto que trabaja y se adentren en el estudio de su subjetividad y su desarrollo, implica profundos cambios de enfoque y no se reduce simplemente a la 
utilización de metodologías cualitativas. Conceptos y cuerpos de conocimientos que den cuenta de la complejidad real del fenómeno deben ser utilizados, concepciones complejas y abrangentes que no simplifiquen y fragmenten el sujeto deben ser introducidas y concepciones epistemológicas que permitan la construcción del conocimiento en su complejidad deben ser utilizadas.

Si bien las investigaciones que tratan de aproximarse a la realidad psicológica de los adolescentes que trabajan no son aún dominantes, resultan interesantes por la utilización de metodologías cualitativas, mostrando, incluso, mucha creatividad en los instrumentos utilizados. Un ejemplo de ello fue el trabajo realizado por Monteiro y Dollinger (1996) con sujetos entre 10 y 15 años participantes del programa Farmacia Viva desarrollado en Fortaleza utilizando pesquisa cualitativa etnográfica y el método de ensayo auto fotográfico. Este tipo de investigaciones evidencia el interés por una aproximación al estudio de aspectos subjetivos de los adolescentes que trabajan, a pesar de que la forma en que se utiliza y se procesa la información en la mayor parte de ellos, responde a una epistemología cuantitativa que limita las posibilidades de avanzar en la comprensión de la diversidad de formas en que la subjetividad es constituida en la situación de trabajo.

Consideramos que el estudio del significado del trabajo en la constitución y el desarrollo de la subjetividad de niños y adolescentes, demanda no sólo la utilización de metodologías cualitativas, sino de concepciones teóricas y epistemológicas que permitan una aproximación mayor a la complejidad de esa realidad psicológica. En ese sentido consideramos que pueden ser útiles los conceptos de subjetividad, personalidad y sujeto, la concepción históricocultural del desarrollo y especialmente la comprensión del desarrollo de la subjetividad como expresión de los contextos sociales e relacionales donde el sujeto está inserido y del sentido subjetivo que estos asumen.

\section{Subjetividad, trabajo y desarrollo}

Las conceptualizaciones a que nos referimos se inscriben dentro del enfoque histórico-cultural del desarrollo humano. Este enfoque, elaborado dentro de la Psicología Soviética a principios del siglo XX y cuyo representante más conocido es L. S. Vygotsky, ha tenido diferentes líneas de desarrollo. Entre ellas, nos interesa especialmente la que ha desarrollado la concepción de subjetividad (Abuljánova,1973; Bozhovich,1976; González Rey, 1985, 1989, 1995, 1997, 2000; González Rey \& Mitjáns Martínez, 1989; Mitjáns Martínez, 2000). Dentro de este marco teórico la categoría subjetividad se refiere a "la organización de los procesos de sentido y significado que aparecen y se organizan de diferentes formas y en diferentes niveles en el sujeto y en la personalidad así como en los diferentes espacios sociales en que el sujeto actúa” (González Rey, 1999, p.108). Esta concepción de subjetividad apunta a la expresión de lo psicológico en su especificidad, su complejidad y su singularidad, tanto a nivel individual como social.

El concepto de subjetividad implica una concepción ontológica. Lo subjetivo es defendido en su propia especificidad constitutiva, considerandose como un segmento de lo real que no es reductible ni explicable a través de ningún outro, a pesar de las estrechas y complejas interrelaciones que tiene con ellos, como, por ejemplo, con lo biológico y con lo social. La especificidad de la subjetividad en su dimensión ontológica significa, esencialmente, que su constitución, organización, funcionamiento y desarrollo, no es explicable en función de las leyes, regularidades y formas de funcionamiento de otros segmentos de la realidad, lo que representa la negación de cualquier tipo de reduccionismo, cuestión que ha estado presente en algunas de las corrientes del pensamiento psicológico (González Rey, 1997). Precisamente, a mi modo de ver, una de las mayores contribuciones que la Psicología puede hacer a la investigación del trabajo infantil es intentar comprender su dimensión subjetiva, lo que implica el reconocimiento de la especificidad anteriormente referida. Si bien el trabajo infantil impacta el desarrollo biológico y las condiciones de escolarización, de salud y en general, de vida de los niños y adolescentes que trabajan, el impacto en la constitución de la subjetividad no puede deducirse directa y linealmente de éstos. La subjetividad, aunque relacionada íntimamente con lo biológico y con lo social, en su especificidad cualitativa tiene mecanismos propios de constitución, funcionamiento y desarrollo cuyo análisis es esencial para la comprensión del impacto del trabajo en su constitución.

El concepto de subjetividad apunta también a la complejidad en que lo psicológico aparece, en sus articulaciones dinámicas, en su carácter procesual y configuracional. Los procesos, contenidos y funciones psicológicas, no existen de forma pura, atomizada y fragmentada sino formando complejas y dinámicas configuraciones donde contenidos y funciones psicológicas de diferente naturaleza y de diferentes niveles de complejidad se articulan y sé ínter penetran. La fragmentación de lo psicológico ha estado presente en muchas de las tendencias dominantes de la Psicología, lo que se expresa hoy en un importante cuerpo de conocimientos con relación a procesos, variables y hechos psicológicos, pero con relativamente poca producción en lo que se refiere a la comprensión del sujeto en su complejidad real. Esta limitación fue claramente expresada por McAdams (1985) cuando afirmó:

\footnotetext{
El estudio científico de la persona total, sin embargo, nunca fue bien integrado dentro de la principal corriente de la Psicología norteamericana en general, ni de la Psicología
} 
de la personalidad en particular (...) Los psicólogos de la personalidad devinieron expertos en extroversión o en necesidad de logro, pero pocos estimaron que sería provechoso ser expertos en personas (p. 21).

Uno de los elementos característicos de la subjetividad es la unidad de cognición e afecto. Capacidades, reflexiones, valores, aspiraciones, necesidades, planes, proyectos, motivaciones, intereses, auto valorización, actividades y relaciones subjetivadas y otros muchos elementos psicológicos complejos se articulan en configuraciones de fuerte valor emocional constituyendo la subjetividad individual. La complejidad de la subjetividad implica también su expresión singular, la forma diferenciada, incluso única e irrepetible, que asume en los individuos concretos. Tendencias dominantes en Psicología prestaron atención a lo general, a lo que aparece como más frecuente y común dentro de los sujetos estudiados, sin focalizar precisamente las formas diferenciadas de su expresión en los sujetos concretos. Con relación al trabajo infantil, la Psicología no debe sólo revelar lo que de psicológico tienen en común niños e adolescentes que trabajan o las formas más frecuentes y visibles del impacto del trabajo en su desarrollo como grupo, sino también, intentar comprender las formas diferenciadas en que esos sujetos son impactados por la situación de trabajo y como se constituyen a partir del significado y sentido que esa realidad tiene para ellos.

La subjetividad, en su conceptualización, incluye a las categorías personalidad y sujeto. La personalidad es entendida, no como un conjunto o sumatoria de rasgos, sino como “configuración sistémica de los principales contenidos y operaciones psicológicas que caracterizan las funciones reguladoras y autorreguladoras del sujeto" (González Rey \& Mitjáns Martínez, 1989, p.16). Por otra parte, la categoría sujeto se refiere a "el individuo concreto, portador de personalidad, quien como características esenciales y permanentes de su condición es actual, interactivo, conciente e intencional" (González Rey, 1995, p. 61). Si la categoría personalidad apunta a formas complejas en que lo psicológico se organiza y representa los aspectos constituidos a lo largo de la trayectoria de vida individual, la categoría sujeto a punta al aspecto interactivo y coyuntural de la subjetividad. Es el sujeto psicológico quien se relaciona con los otros en los contextos sociales donde está inserido, vivencia emociones, toma decisiones y construye representaciones de la realidad. Es en esta condición activa e interactiva que se constituye en un elemento de su propio desarrollo (González Rey, 1995). La mayoría de los niños y adolescentes que trabajan sé inseren en la situación de trabajo con recursos psicológicos configurados en formaciones de la personalidad, recursos que se han constituido y desarrollado a lo largo de su historia de vida anterior. Igualmente, la mayoría de ellos, son sujetos psicológicos en la medida que se han ido constituyendo como tales en función de esa trayectoria.

¿Cómo el hecho de trabajar, de estar inseridos en un contexto supuestamente adulto y no sólo en los contextos esperados de acuerdo a la edad (familiar y escolar, fundamentalmente) puede impactar el desarrollo de la personalidad y de la condición de sujetos de esta población? Éste es un problema, que por su complejidad constituye un desafío para la Psicología y demanda la utilización y producción de conocimientos referidos al desarrollo de la subjetividad. Es en este sentido que el enfoque histórico-cultural del desarrollo psicológico y dentro de él, la línea teórica que ha trabajado la concepción de subjetividad nos parece particularmente prometedora. El enfoque histórico-cultural, sin desconocer el papel que lo biológico puede tener en el desarrollo, privilegia el papel de lo social, lo histórico y lo cultural en constitución de los procesos psicológicos específicamente humanos. De esta forma, lo psicológico, en sus formas más avanzadas de expresión, no es inherente a la naturaleza humana, sino que existe y se constituye en función de la inserción del individuo en contextos culturales y sociales concretos. La línea teórica que desarrolla la concepción de subjetividad dentro de ese enfoque, retoma conceptos importantes como el de situación social del desarrollo (Bozhovich, 1976) y el del papel de lo emocional en el desarrollo de la subjetividad (Bozhovich, 1976; González Rey, 1995, 2000) y desarrolla el concepto de fuerzas motrices del desarrollo de la personalidad identificando tres: la comunicación, las unidades subjetivas de desarrollo y las contradicciones (González Rey, 1995). De hecho el desarrollo de la subjetividad constituye una línea de investigación abierta no sólo al trabajo empírico, sino, fundamentalmente a la elaboración de las conceptualizaciones necesarias para comprender los mecanismos y condiciones que participan en la constitución y desarrollo de la personalidad y de la condición de sujeto.

Cuando nos referimos a la constitución y al desarrollo de la personalidad nos estamos refiriendo al proceso de desarrollo y articulación compleja de los elementos psicológicos que la integran, lo que se expresa en cambios cualitativos en la compleja red de configuraciones de sentido y significado en que estos elementos se organizan y funcionan.

La formación de nuevas necesidades y motivos, la constitución y el desarrollo de la identidad, del sistema de valores, de las aspiraciones, de la concepción del mundo, del sentido de la vida etc., son formas de expresión del desarrollo de la personalidad.

Como anteriormente mencionamos, la constitución y el desarrollo de la subjetividad se da a partir de las interacciones que el niño establece en sus diferentes contex- 
tos culturales de actuación a partir, esencialmente, de los sistemas de comunicación que caracterizan esos contextos (González Rey, 1995; González Rey \& Mitjáns Martínez, 1989). Las vivencias emocionales que se van generando en esos sistemas relacionales resultan decisivas para la constitución de los significados y sentidos que constituyen la subjetividad (González Rey, 1995). En el curso de su historia de vida, el individuo está inserido simultáneamente en contextos diferentes y en cada uno de ellos participa de sistemas de comunicación altamente diferenciados, lo que contribuye a explicar el carácter único e irrepetible de las subjetividades individuales. Éstas constituyen um producto de esas complejas y diversas redes de interacciones, en las cuales el individuo participa de forma cada vez más activa, en la medida que va constituyéndose como sujeto psicológico participando así, de su propia constitución. La subjetividad no es apenas un producto de la conjunción de lo "social" y lo "biológico"; las complejas configuraciones que la integran resultan de las formas de interacción concretas y diferenciadas en las que el individuo participa en sus múltiples y simultáneos contextos de actuación, proceso en el cual, la propia subjetividad en la medida que se constituye, tiene un papeldecisivo.

En el marco conceptual anterior, la situación de trabajo, constituye para los niños y adolecentes trabajadores un contexto adicional de acción e interrelaciones que participa, junto com los otros contextos en que está inserido, en la constitución y en el desarrollo de su subjetividad. Sin contar que, para muchos niños y adolescentes sin estructuras familiares reales y sin la posiblidad de asistir a la escuela por su propia condición de trabajadores, se constituye en el espacio fundamental de su constitución y desarrollo subjetivos. Corresponde precisamente a la Psicología revelar el papel del trabajo en esta dimensión, abordando el problema no a partir de la consideración de un sujeto genérico, sino del sujeto psicológico concreto y de las formas altamente diferenciadas de constitución de su subjetividad.

La Psicología se ha interesado, de diversas formas, en comprender el papel del trabajo en la constitución de lo psicológico. En el desarrollo filogenético el trabajo ha sido considerado como la actividad generadora de las condiciones que posibilitan explicar el salto cualitativo del psiquismo animal al psiquismo humano (Leontiev, 1973, 1980). También el trabajo ha sido ampliamente estudiado como importante factor en la constitución de la identidad (Codo, Sampaio \& Hitomi, 1994; Jacques, 1996, entre otros) y como espacio de satisfacción o de sufrimiento psíquico (por ejemplo, Codo, 1996, 1999; Codo, Sampaio \& Hitomi, 1994; Dejours, 1987; Lima, 1996). Incluso, en los estudios relativamente recientes sobre el desarrollo psicológico en la edad adulta, la actividad laboral, junto a la relación de pareja, ha sido uno de los aspectos sociales más focalizados como se expresa en los trabajos de Wrightsman (1988) y de Rice (1997).

El reconocimiento de la importancia del trabajo en la humanización del hombre y en su desarrollo adulto, constituye razón que se suma a la fundamentación de la necesidad de comprender su impacto en la constitución y en el desarrollo de la subjetividad de los niños y adolescentes que trabajan.

Cuando se analizan las consecuencias negativas del trabajo infantil para el desarrollo psicológico comúnmente se destaca el hecho de que el trabajo infantil impide el tránsito normal por etapas necesarias para el desarrollo cognitivo, afectivo e social (Lopes, 1986; Marques, 1996; Sampaio, Ruiz \& Borsi, 1999, por ejemplo), lo que podría remitir a pensar en una concepción "natural" y por etapas del desarrollo infantil.

A nuestro modo de ver, desde el punto de vista de la constitución y el desarrollo de la subjetividad, la gravedad del trabajo infantil radica en que limita y prácticamente imposibilita, la participación de los pequeños trabajadores en espacios relacionales supuestamente más favorables para el desarrollo de recursos subjetivos deseables como el espacio familiar y especialmente el espacio escolar, espacios donde también lo lúdico, elemento considerado esencial en el desarrollo infantil (Vygotsky, 1960/1979), adquiere formas privilegiadas de expresión.

Indiscutiblemente la escuela, como espacio de adquisición no sólo de conocimientos y capacidades, sino como espacio de socialización, de contacto con la producción cultural humana en su sentido más amplio y de interacciones sociales múltiples, incluyendo a personas de mayor experiencia, constituye un importantísimo espacio de desarrollo que le es robado, precozmente, a los pequeños trabajadores.

La importancia de la escolarización temprana no radica apenas en lo que significa en términos de conocimientos y habilidades necesarias para una inserción efectiva en el mercado de trabajo y en la vida social adulta; realmente su sentido en el desarrollo es mucho más profundo. $\mathrm{Al}$ apropiarse del sistema de conocimientos científicos a través de la actividad escolar, el niño trasciende el nivel de conocimiento espontáneo de la realidad y, como fue expuesto por Vygotsky (1934/1968), tiene la posibilidad de desarrollar formas complejas de actividad pensante, y de hecho, nuevas operaciones mentales. Tiene, también, en ese espacio social estructurado, la posibilidad de desarrollar innumeras capacidades y a partir de los sistemas de comunicación en que participa y de las vivencias emocionales que experimenta, desarrollar valores, motivos, formas de ver el mundo, características funcionales y muchos otros elementos psicológi$\cos$, que se configuran en la subjetividad. 
Hablamos en términos de posibilidades porque ningún espacio social per se es responsable por el desarrollo de unos u otros elementos de la subjetividad. Éstos se constituyen a partir de la compleja conjunción de las características especificas de la multiplicidad de los espacios sociales en que el individuo participa, de los sentidos subjetivos que estos asumen para él y de las vivencias emocionales experimentadas en ellos. Por eso, infelizmente, debido a sus características concretas y al sentido subjetivo que asume, para muchos niños, la escuela no contribuye a desarrollar elementos subjetivos deseables. Para muchos niños y adolescentes la escuela deviene un espacio social que contribuye a la constitución y desarrollo de la inseguridad, la baja autoestima, la forma pasivo-reproductiva de enfrentamiento con la realidad y muchos otros elementos psicológicos que se configuran en su subjetividad.

Enfatizamos que ningún contexto, por muy importante que se muestre para el desarrollo, lo garantiza en términos de recursos subjetivos deseables. Además, su impacto, es altamente diferenciado con relación a los sujetos concretos. El impacto que un determinado contexto tiene para el desarrollo de la subjetividad dependerá de sus características y modos de funcionamiento, pero también de las características subjetivas desarrolladas anteriormente por el sujeto que se insiere en ese contexto y muy especialmente del sentido que esa inserción y ese contexto tienen para él. En relación con esto González Rey (1999) afirma:

El carácter que una influencia tendrá para el desarrollo humano dependerá mucho de su sentido subjetivo, el cual no estará contenido en la influencia en sí misma como un atributo universal, sino que aparece como resultado de la compleja interacción entre la experiencia concreta del sujeto y los recursos subjetivos de que dispone en el momento de experimentarla (p. 111).

Dentro de esta concepción, como mencionamos anteriormente, para los niños y adolescentes que trabajan, el trabajo deviene un contexto relacional que participa de la constitución de su subjetividad en articulación con los otros contextos donde simultáneamente los pequeños trabajadores participan. El trabajo participa de la constitución y desarrollo de la subjetividad, no simplemente como actividad que demanda el desarrollo de determinados conocimientos y competencias, aunque sean mínimos, sino como contex to de actuación e interrelaciones donde el sujeto actúa, se relaciona con otros y experimenta vivencias emocionales específicas. Las características y las exigencias de la actividad laboral concreta, la valoración social de esa actividad, las expectativas que tienen los adultos que los introducen en el mundo del trabajo, las interacciones con los otros en ese contexto específico, los modelos que encuentra, etc., son algunos de los elementos que, como parte de la situación de trabajo, participan en la constitución de la subjetividad. Por otra parte, es necesario insistir en que lo niños y adolescentes al iniciar la actividad laboral ya tienen recursos subjetivos constituidos que se constituyen en un importante mediatizador del impacto de la situación de trabajo en su constitución y desarrollo subjetivo. El significado que el sujeto le confiere al trabajo, el sentido que para él tiene el hecho de tener que trabajar, las necesidades y motivos que se satisfacen en la actividad de trabajo o en las relaciones que establece en ese contexto, las contradicciones que vivencia, los deseos que tiene, las reflexiones que hace y cómo se relaciona con la situación, son parte de los elementos subjetivos que condicionan el impacto del trabajo en la constitución de la subjetividad.

También, el éxito o no que tiene en el ejercicio de la actividad laboral, la valoración que hacen los adultos de sus resultados, la percepción de las nuevas posibilidades que se abren, o de las que se cierran por estar inserido en ese tipo de actividad, las relaciones afectivas que puede encontrar o perder por estar en ese medio, constituyen elementos posibles generadores de emociones, reflexiones y, consecuentemente, de nuevos significados y sentidos subjetivos.

Por lo anteriormente expuesto, consideramos que la complejidad del proceso de constitución de la subjetividad, no permite hacer generalizaciones simplificadas sobre el impacto que en ella tiene el trabajo infantil. Por eso defendemos la necesidad de fortalecer esta línea de investigación que consideramos prometedora en el universo de las contribuciones de la Psicología al estudio de esta problemática.

\section{Un estudio exploratório a partir de la perspectiva de la subjetividad}

Utilizando las concepciones teóricas presentadas anteriormente, hemos comenzado a desarrollar estudios exploratórios con la participación de estudiantes del Curso de Psicología del Centro Universitario de Brasilia que se encuentran en los últimos semestres de su formación. Uno de ellos, que constituyó la Monografía de culminación de estudios de la alumna Maria Raquel Cueto, ejemplifica com clareza los desafios y las perspectivas de un enfoque de esta naturaleza.

Fueron estudiados 10 trabajadores ambulantes, en edades comprendidas entre 10 y 13 años, 9 del sexo masculino y 1 del femenino. Trabajaban como limpiadores de zapatos (2), cuidadores de carro (2), vendedores de golosinas (4) y vendedores de churrasco (2). Con la intención de comenzar a explorar el impacto del trabajo en la constitución de la subjetividad a partir de la perspectiva teórica presentada aquí, nos propusimos, inicialmente, explorar el sentido que la actividad de trabajo tenía para esos sujetos, teniendo en cuenta, como ya hicimos referencia anteriormente, que los 
sentidos subjetivos constituyen un importante elemento para comprender los processos de constitución y desarrollo de la subjetividad. Para ello fueron realizados estudios de caso a partir de la realización de entrevistas semiestructuradas y de la observación de los sujetos en sus actividades e interacciones en el propio ambiente de trabajo.

El proceso de análisis de la información fué realizado dentro de los principios de la epistemologia cualitativa (González Rey, 1993, 1997), donde el investigador tiene un papel activo y utiliza la información en un proceso constructivo tendiente a aproximarse a la caracterización del fenómeno estudiado en su complejidad.

Lo más relevante, a los fines del análisis que estamos realizando aquí, es que, a partir de los estudios de casos realizados, pudieron identificarse cuatro formas diferenciadas de aproximación de esos sujetos al mundo del trabajo, formas diferentes en que significan y vivencian su condición de trabajadores. De forma resumida estas son:

Grupo1: Los sujetos parecen haberse adaptado a la necesidad de trabajar de una forma tranquila, natural. Aparentemente se sienten felices e gratificados por estar trabajando, pues se pueden mantener y al mismo tiempo auxiliar en la manutención familiar, además de sentir que están siendo reconocidos en la esfera social y familiar (Cueto, 2000, p. 24).

Llama la atención que para estos sujetos el trabajo está asociado a vivencias emocionales positivas, en la medida en que satisface necesidades subjetivas de ayuda y compromiso familiar, ya desarrolladas en ellos y a la vez, satisface necesidades de valorización y reconocimiento, fundamentalmente asociadas a la esfera familiar donde mantienen un vinculo afectivo y estructurado estable.

Grupo 2: Los sujetos, que comenzaron a trabajar para contribuir a las finanzas familiares, parecen haberse conformado con la necesidad de trabajar, pero si pudieran escoger, les gustaria tener más tiempo para dedicarse al estudio y a los juegos. Estudiar parece adquirir gran significación para estos adolescentes ya que a través del estudio esperan satisfacer sueños y necesidades y conseguir una vida mejor y más digna. (Cueto, 2000, p. 25).

En estos casos las vivencias experimentadas con relación a la actividad de trabajo son contradictorias ya que estos sujetos poseen otros motivos constituidos que entran en contradicción con la actividad de trabajo, especialmente el estudio, al que perciben como la vía idónea para alcanzar realizaciones futuras. A pesar de esto valoran y vivencian como aspecto positivo de su condición de trabajadores la posibilidad de ayudar a la familia y de ser importantes para ella.

Grupo 3: Los sujetos comenzaron a trabajar espontáneamente motivados por el "bienestar" material que el dinero les podía ofrecer. No tienen que ayudar al mantenimiento familiar, pudiendo quedarse con todo el dinero que recaudan. En ese sentido se sienten valorizados e más independientes. Al mismo tiempo, reconocen las desigualdades sociales e se sienten excluidos y marginalizados. Parece que ven el dinero como una especie de "pasaporte" para la aceptación social y la búsqueda de una vida mejor. Reconocen la importancia de estudiar pero el estudio no los motiva ya que la escuela no les trae refuerzos inmediatos, ni satisface la necesidad de aceptación social que poseen. (Cueto, 2000, p. 25)

El trabajo adquiere un sentido positivo para estos sujetos, fundamentalmente como espacio de independencia material y psicológica. Otros motivos, como el estudio, no aparecen como motivos actuantes o ocupan una posición totalmente subordinada en la jerarquía motivacional en desarrollo. El trabajo constituye una configuración subjetiva importante que incluye sus perspectivas de futuro, y mobiliza con fuerza su acción presente.

Grupo 4: Los sujetos no comprenden muy bien porque tienen que trabajar. Visiblemente la necesidad de trabajar los incomoda y los deja insatisfechos. Sienten mucho la falta de poder practicar actividades infantiles como jugar $y$ estudiar, además de sentirse cansados con la rutina de trabajo y estudio. Estos sujetos demostraron necesidades lúdicas muy acentuadas y carencia afectiva. (Cueto, 2000, p. 25)

En estos sujetos el trabajo genera, esencialmente, vivencias emocionales negativas y es fuente permanente de insatisfacción. Es significado como una actividad adulta que debe ser ejercida por otros, adquiriendo un sentido profundamente negativo, en la medida en que no es espacio de satisfacción de importantes necesidades subjetivas constituídas en ellos como lo es la necesidad de afecto, rechazando de forma profunda a los adultos que les imponen el trabajo como forma de vida.

Vemos cómo el rescate del sujeto en la investigación de las consecuencias del trabajo infantil permite evidenciar formas muy diversas en que éste se subjetiviza. Esta subjetivación diferenciada del trabajo, permite hipotetizar que éste tendrá también influencias diferenciadas en la constitución y en el desarrollo posterior de la subjetividad de estos sujetos. El trabajo, presumiblemente, marcará trayectorias diferentes de desarrollo en función del significado y sentido que asume en la subjetividad y su impacto en el desarrollo subjetivo no será el mismo, por ejemplo, en aquellos sujetos en que se configura como fuente de satisfacción de necesidades subjetivas importantes, que en los sujetos que lo vivencian como espacio de constante insatisfacción.

Esta línea de investigación permite cuestionar el carácter absoluto que asumen ciertas afirmaciones sobre las 
consecuencias psicológicas del trabajo infantil tales como "comprometimiento del desarrollo físico, cognitivo y emocional, cancelamiento de proyectos de vida y deseestructuración del mundo infantil: el mundo de libertad se convierte en le mundo del deber (Ferreira, Silva \& Hoelzel, 2000). Si bien estas afirmaciones son válidas con relación al desarrollo de muchos niños y adolescentes que trabajan, no necesariamente ejemplifican el papel que la situación de trabajo tiene en el desarrollo de otros. Los sujetos del grupo 3 por ejemplo, precisamente desarrollan sus proyectos de vida en su relación con el espacio de trabajo, que además es vivenciado como un espacio de libertad, bien diferente a la percepción que tienen de la escuela que es significada como un espacio de deber y de exclusión psicológica.

Comprender las formas complejas y diversas en las que el trabajo participa como espacio de constitución de la subjetividad es esencial para el diseño de acciones educativas y de orientación diferenciadas, que realmente puedan resultar efectivas en función de su adecuación a los procesos y mecanismos que caracterizan de forma concreta el funcionamiento subjetivo de los sujetos a los que van dirigidas. Construir y desarrollar esas estrategias diferenciadas, ya sea para el trabajo directo con los niños y adolescentes que trabajan o para el trabajo con los adultos que interactúan con ellos (familiares, profesores etc.) constituye un desafío adicional que es necesario enfrentar por la significación que pueden tener para su futuro desarrollo.

\section{Referências bibliográficas}

Abuljánova, K. A (1985). El sujeto de la actividad psíquica. México: Roca. Bonamigo, L. D. (1996). O trabalho na construção da identidade: um estudo sobre meninos trabalhadores na rua. Reflexão e Crítica, 9, 123130.

Bozhovich, L. I. (1976). La personalidad y su formación en la edad infantil. La Habana: Pueblo y Educación.

Campos, H., Dimenstein, M., \& Francischini, R. (2000). Trabalho infantojuvenil: panorama geral e perspectivas de investigação/intervenção [Resumo]. In Sociedade Brasileira de Psicologia (Org). XXX Reunião Anual de Psicologia. Resumos. (p. 58). Ribeirão Preto.

Codo, W. (1996). Um diagnóstico do trabalho (Em busca do prazer). In A Tamayo, J. E Borges-Andrade, W. Codo (Orgs.), Trabalho, organizações e cultura (Vol 11, pp. 36-55) Riberão Preto: Coletâneas da ANPEPP.

Codo, W. (Org). (1999). Educação: carinho e trabalho. Petrópolis: Vozes.

Codo, W., Sampaio, J. J. C., \& Hitomi, A. H. (1994). Indivíduo, trabalho e sofrimento. Petrópolis: Vozes.

Cueto, M. R. R. (2000). O impacto do trabalho na subjetividade infantil. Monografia de final de Curso. Não publicada. Curso de Psicología. Brasília: Centro Universitário de Brasília, Brasília.

Dejours, C. (1987). A loucura do trabalho. São Paulo: Oboré.

Ferreira, M., Silva, R., \& Hoelzel, F. (2000). O trabalho precoce e a construção de um diálogo necessário: uma reflexão sobre a articulação entre produção científica e sociedade com vistas ao aprimoramento das políticas públicas voltadas para a infância e a adolescência em cenários de desenvolvimento. [Resumo] In Sociedade Brasileira de
Psicologia (Org.), XXX Reunião Anual da Sociedade Brasileira de Psicologia. Resumos (p. 59). Ribierão Preto: SBP.

Garcia Rosas, L. F. (1973). Psicologia: um espaço de dispersão do saber. Radice, 4, 20-26.

González Rey, F. (1985). Psicologia de la personalidad. La Habana: Pueblo y Educación.

González Rey, F. (1989). Psicología: principios y categorías. La Habana: Ciencias Sociales.

González Rey, F. (1993). Problemas epistemológicos de la Psicología. México: UNAM

González Rey, F. (1995). Comunicación, personalidad y desarrollo. La Habana: Pueblo y Educación.

González Rey, F. (1997). Epistemología cualitativa y subjetividad. São Paulo: EDUC.

González Rey, F. (1999). Psicologia e Educação: desafíos e projeções. In O. A. Rays (Org), Trabalho pedagógico: realidades e perspectivas (pp 102-117). Porto Alegre: Sulina.

González Rey, F. (2000). O emocional na constituição da subjetividade. In S. Lane \& Y. Araújo (Org.), Arqueologia das emoções (pp. 36-56). Petrópolis: Vozes.

González Rey, F., \& Mitjáns Martínez, A (1989). La personalidad: su educación y desarrollo. La Habana: Pueblo y Educación.

Jaques, M. G. C. (1996). Identidade e trabalho: uma articulação indispensável. In A. Tamayo, J. E.Borges-Andrade \& W. Codo (Orgs.), Trabalho, organizações e cultura (vol. 11, pp. 21-26). Ribeirão Preto.: ANPEPP.

Leontiev, A. N. (1973). El Hombre y la cultura. México: Grijalbo.

Leontiev, A. N. (1980). O desenvolvimento do psiquismo. São Paulo: Moraes.

Lima, M. E. A. (1996). A pesquisa em saúde mental e trabalho. In A. Tamayo, J. E. Borges-Andrade \& W. Codo (Orgs.), Trabalho, organizações e cultura (Vol 11, pp. 27-35) Ribeirão Preto: ANPEPP.

Lopes, V. L. S. (1986). Adolescência e criatividade: o trabalho precoce e suas relações com a personalidade. Arquivos Brasileiros de Psicologia, 38, 95-114.

Marques, W. E. U. (1996). Crianças e adolescentes marginalizados: de como a rua passou a ser este lugar. Trabalho \& Educação. NETE, Q 149-168.

Marques, W. E. U. (1998). Criança trabalhadora, família e identidade: desafíos para o mundo actual. Trabalho \& Educação. NETE, 3143159.

McAdams, D. P. (1985). Power, intimacy and the live story: Personological inquires into identity. Illinois: Darsey Press.

Mitjáns Martínez, A. (2000). Criatividade, personalidade e educação. Campinas: Papirus.

Monteiro, J. M. C., \& Dollinger, S. J. (1996). Estudo etnográfico e autofotográfico do menino na rua em Fortaleza, Ceará. In S. H. Koller (Org.), Aplicações da Psicologia na melhoria da qualidade de vida (vol. 12, pp. 45-60). Porto Alegre: ANPEPP.

Rice, F. P. (1997). Desarrollo humano: Estudio del ciclo vital. México: Prentice Hall.

Sampaio, J. J. C., Ruiz, E. M., \& Borsi, I. C. F. (1999). Trabalho precoce e psiquismo infantil. In J. J. C. Sampaio, E. M. Ruiz \& I. C. F. Borsi (Orgs.). Trabalho, saúde e subjetividade (pp. 25-31). Fortaleza: INESP/ EDUECE.

Vygotsky, L. S. (1968). Pensamiento y lenguaje. La Habana: Edición Revolucionaria. (Texto original publicado en 1934).

Vygotsky, L. S. (1979). El desarrollo de los procesos psicológicos superiores. Barcelona: Crítica (Texto original publicado en 1960)

Wrightsman, L. S. (1988). Personality development in adulhood. California: Sage. 
244 A. Mijtáns Martinez

Notas

1 A los fines de este trabajo, utilizaremos la expresión tradicional de "trabajo infantil" como equivalente a las de "trabajo infanto-juvenil" y "trabajo precoz".

Albertina Mitjáns Martinez, doutora em Psicologia pela Universidad de La Habana (Cuba), é Pesquisadora Associada Senior do Instituto de Psicologia da Universidade de Brasília e professora do Centro Universitário de Brasília e do Instituto de Educação Superior de Brasília, Brasília (DF). Endereço para correspondência: SQS 407, Bloco R, apto. 206. 70256-180, Brasília, DF. Telefax: (61) 244.6753. E-mail: amitjans@mymail.com.br. 